\title{
Optimization Algorithms for Material Pyrolysis Property Estimation
}

\author{
CHRIS LAUTENBERGER, and CARLOS FERNANDEZ-PELLO \\ Department of Mechanical Engineering \\ University of California at Berkeley \\ Berkeley, CA 94720 USA
}

\begin{abstract}
This paper critically assesses the experimental tools and optimization techniques that can be applied to determine material pyrolysis properties intended for fire modeling. It is argued that while independent measurement of material pyrolysis properties using multiple specialized laboratory tests may be the most fundamentally correct way to determine these properties, due to practical considerations optimization offers definite advantages for fire modeling and will likely remain an integral part of material pyrolysis property estimation. The performance of four optimization algorithms that have been implemented in Gpyro is assessed in terms of efficiency (how quickly it converges to a solution) and accuracy (how close the converged solution is to the global optimum) by extracting 19 material pyrolysis properties from a set of synthetic cone calorimeter data. Widely-used genetic algorithm optimization techniques perform poorly in comparison to the shuffled complex evolution (SCE) algorithm, recently applied to material pyrolysis property estimation by Chaos et al. It is shown that SCE consistently converges to the same solution and is capable of reproducing material pyrolysis properties within $\sim 1 \%$ of the actual values used to generate the synthetic data set. This work suggests that SCE is capable of determining a unique set of material pyrolysis properties that correspond to the globally optimal solution.
\end{abstract}

KEYWORDS: pyrolysis, material properties, property estimation.

\section{NOMENCLATURE LISTING}

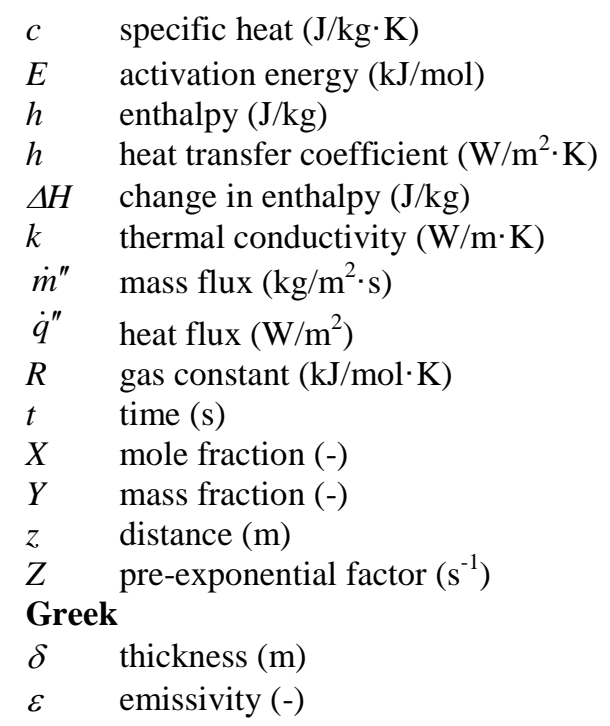

$\begin{array}{ll}\rho & \text { density }\left(\mathrm{kg} / \mathrm{m}^{3}\right) \\ \dot{\omega}^{\prime \prime \prime} & \text { reaction rate }\left(\mathrm{kg} / \mathrm{m}^{3} \cdot \mathrm{s}\right) \\ \text { subscripts } \\ \infty & \text { ambient } \\ 0 & \text { initial } \\ 1 & \text { reaction } 1 \\ 2 & \text { reaction } 2 \\ A & \text { species A } \\ B & \text { species B } \\ c & \text { convective } \\ C & \text { species C } \\ e & \text { external } \\ d & \text { destruction } \\ f & \text { formation } \\ g & \text { gas } \\ p & \text { pressure } \\ v & \text { volatilization }\end{array}$

\section{INTRODUCTION}

Since 2006, at least three comprehensive pyrolysis models (FDS [1], Thermokin [2], and Gpyro [3]) have been developed within the fire community and broadly disseminated. Despite independent development of these three models by separate groups, their mathematical and numerical formulations are quite similar. These three models provide nearly identical solutions when provided with matching material properties, with minor solution differences attributed to details of certain submodels (internal radiation, reaction 
treatment, etc.). On this basis, it seems that consensus has been reached regarding the appropriate overall framework and mathematical formulation to simulate material decomposition and burning via comprehensive pyrolysis modeling. However, there is currently no consensus regarding how to appropriately apply various tools and techniques to determine the material pyrolysis properties that these pyrolysis models require as input to simulate a material's fire performance.

Recognizing this deficiency, the US National Institute of Standards and Technology (NIST) has commissioned Worcester Polytechnic Institute (WPI), Southwest Research Institute (SwRI), and the Society of Fire Protection Engineers (SFPE) to develop the SFPE Engineering Guide for Estimating Material Pyrolysis Properties for Fire Modeling [4]. Once published, this Guide will provide badly-needed clarity and guidance in this area. The overall philosophy of the SFPE Guide currently under development can be summarized as "measure the material pyrolysis properties that you can, and estimate the rest using optimization".

Several of the required material pyrolysis properties can in principle be measured independently using specialized laboratory experiments. Fundamentally, this is the purest way to determine the required material pyrolysis properties. However, due to practical considerations that will be discussed later, measurement of independent material pyrolysis properties using multiple specialized laboratory experiments will likely be of limited use for fire modelers outside of a research environment.

For this reason, it is expected that optimization will play an increasingly important role in material pyrolysis property estimation. In the context of the SFPE Guide and material pyrolysis property estimation in general, optimization refers to the process of solving an inverse problem. By working backward from a set of experimental data (e.g., mass loss rate and temperature measurements from the cone calorimeter), a set of 'equivalent properties' can be determined by finding the input parameters that best reproduces that experimental data set when provided as input to a pyrolysis model. This optimization can be accomplished using several techniques, ranging from manual optimization to automated massively parallel search techniques such as genetic algorithms.

\section{MATERIAL PYROLYSIS PROPERTIES}

'Material pyrolysis properties' may mean different things in different contexts. Therefore, a simplified 1-D mathematical model of material heating and pyrolysis is presented below, and various material pyrolysis properties are then identified. Since this is difficult to do in a generalized way, a typical charring material with two reactions and three condensed phase species is used as an example. Other simplifications include:

- Shrinkage or swelling (volume change) is negligible

- Thermophysical properties are independent of temperature

- In-depth absorption of radiation is negligible

- Radiation heat transfer across pores is negligible

- Gas phase and condensed phase are in thermal equilibrium

- Two-step serial reaction mechanism with three condensed-phase species

This two-step, three-component formulation is prototypical of wood: when wood is heated, a carbonaceous char is formed, and under additional heating (particularly in an oxidative environment) this char may further react to form ash or residue. For generality, the three condensed-phase components will be referred to as $A, B$, and $C$. One reaction converts $A$ to $B$ plus gas, and a second reaction converts $B$ to $C$ plus gas:

$$
A \rightarrow B+\text { gas } \quad ; \quad B \rightarrow C+\text { gas }
$$

Due to the creation and destruction of species $A, B$, and $C$ by the reactions in Eq. 1, separate conservation equations must be solved to determine their local mass fractions:

$$
\frac{\partial\left(\bar{\rho} Y_{A}\right)}{\partial t}=-\dot{\omega}_{d A}^{\prime \prime \prime} \quad ; \quad \frac{\partial\left(\bar{\rho} Y_{B}\right)}{\partial t}=\dot{\omega}_{f B}^{\prime \prime \prime}-\dot{\omega}_{d B}^{\prime \prime \prime} \quad ; \quad \frac{\partial\left(\bar{\rho} Y_{C}\right)}{\partial t}=\dot{\omega}_{f C}^{\prime \prime \prime}
$$


Where $\dot{\omega}_{d A}^{\prime \prime \prime}$ is the destruction of species $A, \dot{\omega}_{f B}^{\prime \prime \prime}$ is the formation of species $B, \dot{\omega}_{d B}^{\prime \prime \prime}$ is the destruction of species $B$, and $\dot{\omega}_{f C}^{\prime \prime \prime}$ is the formation of species $C$.

Since the condensed phase consists of three separate components $(A, B$, and $C)$, it is necessary to define the following 'averaged' quantities that appear in the conservation equations and boundary conditions:

$$
\begin{aligned}
& \bar{k}=X_{A} k_{A}+X_{B} k_{B}+X_{C} k_{C} \\
& \bar{\rho}=X_{A} \rho_{A}+X_{B} \rho_{B}+X_{C} \rho_{C} \\
& \bar{c}=Y_{A} c_{A}+Y_{B} c_{B}+Y_{C} c_{C} \\
& \bar{\varepsilon}=X_{A} \varepsilon_{A}+X_{B} \varepsilon_{B}+X_{C} \varepsilon_{C}
\end{aligned}
$$

Where $X$ denotes condensed-phase volume fraction and $Y$ denotes condensed-phase mass fraction. Here, we see the appearance of twelve material pyrolysis properties: $k_{A}, k_{B}, k_{C}, \rho_{A}, \rho_{B}, \rho_{C}, c_{A}, c_{B}, c_{C}, \varepsilon_{A}, \varepsilon_{B}$, and $\varepsilon_{C}$. Of these, only $\rho_{A}$ (and possibly $\rho_{C}$ ) can be easily measured.

The destruction rate of Species $A$ and $B$ are modeled as Arrhenius reactions:

$$
\dot{\omega}_{d A}^{\prime \prime \prime}=\left(\frac{\bar{\rho} Y_{A}}{\left(\bar{\rho} Y_{A}\right)_{\Sigma}}\right)^{n_{1}}\left(\bar{\rho} Y_{A}\right)_{\Sigma} Z_{1} \exp \left(-\frac{E_{1}}{R T}\right) ; \quad \dot{\omega}_{d B}^{\prime \prime \prime}=\left(\frac{\bar{\rho} Y_{B}}{\left(\bar{\rho} Y_{B}\right)_{\Sigma}}\right)^{n_{2}}\left(\bar{\rho} Y_{B}\right)_{\Sigma} Z_{2} \exp \left(-\frac{E_{2}}{R T}\right)
$$

Equation 4 introduces the material pyrolysis properties $E, Z$, and $n$ that characterize the decomposition kinetics of each reaction. Each of these three parameters must be determined for each reaction, so six kinetic parameters must be determined for the specific case considered here.

The formation rate of Species $B$, Species $C$, and gases can be calculated from reactant/product density ratios as:

$$
\dot{\omega}_{f B}^{\prime \prime \prime}=\frac{\rho_{B}}{\rho_{A}} \dot{\omega}_{d A}^{\prime \prime \prime} \quad ; \quad \dot{\omega}_{f C}^{\prime \prime \prime}=\frac{\rho_{C}}{\rho_{B}} \dot{\omega}_{d B}^{\prime \prime \prime} \quad ; \quad \dot{\omega}_{f g}^{\prime \prime \prime}=\left(1-\frac{\rho_{B}}{\rho_{A}}\right) \dot{\omega}_{d A}^{\prime \prime \prime}+\left(1-\frac{\rho_{C}}{\rho_{B}}\right) \dot{\omega}_{d B}^{\prime \prime \prime}
$$

Condensed-phase mass conservation states that the local rate of change in condensed-phase density is equal to the local volumetric rate of gas formation $\left(\dot{\omega}_{f g}^{\prime \prime \prime}\right)$ :

$$
\frac{\partial \bar{\rho}}{\partial t}=-\dot{\omega}_{f g}^{\prime \prime \prime}
$$

For the case where gaseous volatiles escape instantaneously to the exterior ambient with no resistance to flow or internal pressure build up, the local gaseous mass flux at any point inside the decomposing solid can be calculated from gaseous mass conservation as:

$$
\dot{m}^{\prime \prime}(z)=\int_{z}^{\delta} \frac{\partial \bar{\rho}}{\partial t} d \zeta=-\int_{z}^{\delta} \dot{\omega}_{f g}^{\prime \prime \prime} d \zeta
$$

where $\delta$ is the sample thickness and the $z$ coordinate increases with depth into the solid, i.e. $z=0$ corresponds to the sample surface and $z=\delta$ corresponds to the sample's back face.

Finally, the condensed phase energy conservation is: 


$$
\begin{aligned}
& \frac{\partial(\bar{\rho} \bar{h})}{\partial t}+\dot{m}^{\prime \prime} c_{p g} \frac{\partial T}{\partial z}=\frac{\partial}{\partial z}\left(\bar{k} \frac{\partial T}{\partial z}\right)-\left(1-\frac{\rho_{B}}{\rho_{A}}\right) \dot{\omega}_{d A}^{\prime \prime \prime} \Delta H_{v o l 1}-\left(1-\frac{\rho_{C}}{\rho_{B}}\right) \dot{\omega}_{d B}^{\prime \prime \prime} \Delta H_{v o l 2} \\
& -\dot{\omega}_{d A}^{\prime \prime \prime} h_{A}+\left(\dot{\omega}_{f B}^{\prime \prime \prime}-\dot{\omega}_{d B}^{\prime \prime \prime}\right) h_{B}+\dot{\omega}_{f C}^{\prime \prime \prime} h_{C}
\end{aligned}
$$

The heat of volatilization $\left(\Delta H_{v o l}\right)$ appearing on the right hand side of Eq. 8 must be determined for each reaction, so Eq. 8 introduces two additional material pyrolysis properties $\left(\Delta H_{v o l 1}\right.$ and $\left.\Delta H_{v o l 2}\right)$.

The boundary and initial conditions on the energy equation are:

$$
\begin{aligned}
& -\left.\bar{k} \frac{\partial T}{\partial z}\right|_{z=0}=\bar{\varepsilon} \dot{q}_{e}^{\prime \prime}-\bar{\varepsilon}\left(\left.T^{4}\right|_{z=0}-T_{\infty}\right)-h_{c}\left(\left.T\right|_{z=0}-T_{\infty}\right) ;-\left.\bar{k} \frac{\partial T}{\partial z}\right|_{z=\delta}=0 \\
& \left.T\right|_{t=0}=T_{0}
\end{aligned}
$$

And the initial conditions on the species conservation equation are:

$\left.Y_{A}\right|_{t=0}=1 \quad ;\left.\quad Y_{B}\right|_{t=0}=0 \quad ;\left.\quad Y_{C}\right|_{t=0}=0$

In summary, 20 material pyrolysis properties appearing in the above equations must be determined: $k_{A}, \rho_{A}$, $c_{A}, \varepsilon_{A}, k_{B}, \rho_{B}, c_{B}, \varepsilon_{B}, k_{C}, \rho_{C}, c_{C}, \varepsilon_{C}, Z_{1}, E_{1}, n_{1}, \Delta H_{v o l 1}, Z_{2}, E_{2}, n_{2}, \Delta H_{v o l 2}$. Of these, only $\rho_{A}$ can be easily measured directly, leaving 19 material pyrolysis properties that must be determined. It can be envisaged that the number of properties that must be determined increases rapidly with the 'complexity' of the material under consideration. For example, if in-depth radiation heat transfer, radiation heat transfer across pores, or temperature-dependent material properties are considered, then the number of material pyrolysis properties that must be determined increases significantly. As the complexity of a modeling approach increases, the number of material pyrolysis properties that must be determined quickly becomes prohibitive. It is for this reason that a key to pyrolysis modeling for practical applications is to strike a balance between accuracy and complexity.

\section{EXPERIMENTAL TECHNIQUES FOR DETERMINING MATERIAL PYROLYSIS PROPERTIES}

In the previous section, the material pyrolysis properties that control a material's overall flammability within the context of a comprehensive pyrolysis model were identified for a prototypical two-reaction, three-component material. For that particular case, 19 material pyrolysis properties must be determined.

Conventional flammability tests such as the cone calorimeter, lateral ignition and flame spread test (LIFT), fire propagation apparatus (FPA), etc. can provide 'effective' values of empirical quantities such as thermal inertia $(k \rho c)$, ignition temperature $\left(T_{i g}\right)$, heat of gasification $\left(L_{g}\right)$, etc. However, these properties do not appear anywhere in Eqs. 1-10 and for that reason these quantities are not useful for comprehensive pyrolysis modeling [1-3] including fire modeling with tools such as Fire Dynamics Simulator (FDS) [1] or FireFOAM [5].

Specialized laboratory experiments, many of them standardized as ASTM test methods, are available to measure several of the material pyrolysis properties identified above. The SFPE Engineering Guide for Estimating Material Pyrolysis Properties for Fire Modeling [4] provides an excellent discussion of the various experimental techniques that are available.

The primary categories of commercially-available instruments that could potentially be applied to measure the necessary material pyrolysis properties are:

- Thermal analysis, i.e. thermogravimetric analysis (TGA), differential scanning calorimetry (DSC), and simultaneous thermal analysis (STA). Material pyrolysis properties determined: $Z, E, n, c$, $\Delta H_{\text {vol }}$. 
- Tests for thermal conductivity (ASTM E1530 guarded heat flow meter - GHFM) or thermal diffusivity (ASTM E1461 laser flash apparatus - LFA). Material pyrolysis properties determined: $k$ (GHFM) or $k / \rho c$ (LFA).

- Hemispherical directional reflectometer (HDR). Material pyrolysis properties determined: $\varepsilon$.

- Pycnometer (Pyc). Material pyrolysis properties determined: $\rho$

- Slab pyrolysis experiments (ASTM E1354 cone calorimeter, ASTM E2058 fire propagation apparatus, etc.)

The material pyrolysis properties that can be measured (M) or inferred (I) from each instrument are indicated in Table 1. For all inferred (I) quantities, some type of regression analysis or optimization technique is necessary to determine the required properties. However, such techniques are not necessary for directly measured $(\mathrm{M})$ quantities.

Table 1. Material pyrolysis properties that can be Measured (M) or Inferred (I) from laboratory experiments: thermogravimetric analysis (TGA), differential scanning calorimetry (DSC), simultaneous thermal analysis (STA), guarded heat flow meter (GHFM), laser flash apparatus (LFA), hemispherical directional reflectometer (HDR), pycnometer (Pyc), cone calorimeter/fire propagation apparatus/similar (Cone/FPA).

\begin{tabular}{|c|c|c|c|c|c|c|c|c|}
\hline Property & TGA & DSC & STA & GHFM & LFA & HDR & Pyc & Cone/FPA \\
\hline$k$ & & & & $\mathrm{M}$ & & & & $\mathrm{I}$ \\
$\rho$ & & & & & & & $\mathrm{M}$ & $\mathrm{I}$ \\
$c$ & & $\mathrm{M}$ & $\mathrm{M}$ & & & & & $\mathrm{I}$ \\
$k / \rho c$ & & & & & $\mathrm{M}$ & & & $\mathrm{I}$ \\
$Z$ & $\mathrm{M} / \mathrm{I}$ & & $\mathrm{M} / \mathrm{I}$ & & & & & $\mathrm{I}$ \\
$E$ & $\mathrm{M} / \mathrm{I}$ & & $\mathrm{M} / \mathrm{I}$ & & & & & $\mathrm{I}$ \\
$n$ & $\mathrm{M} / \mathrm{I}$ & & $\mathrm{M} / \mathrm{I}$ & & & & & $\mathrm{I}$ \\
$\Delta H_{\text {vol }}$ & & $\mathrm{M}$ & $\mathrm{M}$ & & & & & $\mathrm{I}$ \\
$\varepsilon$ & & & & & & $\mathrm{M}$ & & $\mathrm{I}$ \\
\hline
\end{tabular}

Two philosophically different approaches to determining material pyrolysis properties emerge:

1. Measure (or measure/infer) as many material pyrolysis properties as possible using specialized laboratory instruments: TGA/DSC/STA, GHFM/LFA, HDR, and pycnometry.

2. Infer all required properties from the cone calorimeter, FPA, or similar experiments using optimization.

In both cases, the 'accuracy' of the material pyrolysis properties should be assessed by comparing optimized model calculations to data from slab-type pyrolysis experiments such as the cone calorimeter or fire propagation apparatus. For Approach \#1 (measure as much as possible) this could be considered a 'quasi-blind' test; but for Approach \#2 (infer via optimization), this is a 'self' test.

Approach \#1 (measure as much as possible) is more scientifically rigorous than Approach \#2 (infer via optimization). It is appropriate for fundamental studies of material flammability or pyrolysis where the goal is to delve into the controlling mechanisms of material flammability or to predict the outcome of smallscale flammability tests (cone calorimeter, UL 94, etc.).

However, independent measurement of material pyrolysis properties presents several challenges. Measuring thermal properties (thermal conductivity, specific heat capacity, and density) at temperatures above which a material begins to decompose (pyrolyze) is problematic due to off-gassing. Similar difficulties are encountered for materials that melt, shrink, or swell when heated. Pyrolysis models require thermal properties of individual components (i.e., Species $A, B$, and $C$ in Eqs. 1-10) but it is not clear how thermal properties of individual components can be determined from experiments that measure thermal properties of a pyrolyzing sample containing unknown mass fractions of different components. Kinetics constants obtained from thermal analysis experiments at typical heating rates of $<1 \mathrm{~K} / \mathrm{s}$ may not apply to 
heating rates of $>100 \mathrm{~K} / \mathrm{s}$ encountered in fires. These measurements are cumbersome to conduct for 'nonsimple' real-world materials (e.g., layered/laminated composites, inhomogeneous/anisotropic materials, sandwich panels, honeycomb core materials, etc.). Due to the error bars on each independently-measured material pyrolysis property, the predictions of a comprehensive pyrolysis model using these material pyrolysis properties may also be subject to considerable uncertainty. Nonetheless, Lattimer and Ouellette [6] and Stoliarov et al. [7,8] demonstrated that independently-measured material pyrolysis properties, when provided as input to a comprehensive pyrolysis model, provide good predictions of slab-type pyrolysis experiments for the materials they investigated.

Approach \#2 (infer via optimization) has been dismissed by some researchers as 'just curve-fitting'. Early studies on optimization for material pyrolysis property estimation pointed out that the problem is ill-posed, meaning that a unique solution does not exist and that multiple sets of different material pyrolysis properties may provide equally good fits to experimental data. This is a valid criticism, but it will be demonstrated later in this paper that improved optimization algorithms are capable of locating a unique set of material pyrolysis properties corresponding to the global optimum. Thus, material pyrolysis properties determined by optimization should be viewed as 'equivalent properties' that provide optimal agreement between model calculations and the experimental data set for the particular set of modeling assumptions that were invoked. These modeling assumptions include the assumed reaction mechanism and decisions on whether or not to include phenomena such as temperature-dependency of thermal properties, in-depth radiation absorption, radiation heat transfer across char pores, shrinkage/swelling, etc. If the cumulative modeling assumptions accurately represent a material's behavior, then the equivalent properties should be true material properties. Therefore, the material pyrolysis properties determined by Approach \#2 are not necessarily less accurate than those determined by direct independent measurement, particularly when one considers the difficulties identified earlier. Furthermore, pyrolysis and combustion of 'non-simple' materials can also be simulated with Approach \#2 using the equivalent properties concept. For practical situations where the goal is to apply fire modeling to predict fire development (i.e., how quickly will a fire grow and how big will it get), Approach \#2 offers significant cost and time savings over Approach \#1. Based on this discussion, it is concluded that for real-world applications of fire growth modeling, Approach \#2 (infer via optimization) offers distinct advantages over Approach \#1 (measure as much as possible).

\section{OPTIMIZATION METHODS FOR INFERRING MATERIAL PYROLYSIS PROPERTIES: AN INVERSE PROBLEM}

Since the late 1990s, several workers have applied optimization techniques to solve inverse problems for material pyrolysis property estimation [9-33]. Some of these contributions are cataloged in Table 2 (not intended to be an exhaustive listing). One of the earliest fire-related efforts is that of de Ris and Yan [9] who, in 1997-98, developed a spreadsheet-based optimization method that determines a set of equivalent properties by maximizing the agreement between the calculations of a linearized version of Kung's pyrolysis model [34] and experimental data obtained from the fire propagation apparatus. In 1999, Kanevce et al. [10] applied a Newton-Raphson/steepest descent method to determine thermal properties, reaction kinetics, and heats of reaction for a phenolic composite under intense heating. One of the first applications of genetic algorithms (GA) to estimate kinetic parameters from thermogravimetric analysis was Şahin et al. [11] in 2001. In 2005, Zhao and Dembsey [12] applied the method of de Ris and Yan [9] to wood and FRP, and Theuns et al. [13] applied a downhill Simplex method to extract particleboard material pyrolysis properties from slab type pyrolysis experiments.

In 2004, Rein and co-workers at UC Berkeley began applying GA to estimate material pyrolysis properties, first from TGA experiments [14,15], and later from slab type pyrolysis experiments such as the cone calorimeter [16]. As can be gleaned from Table 2, GA has become the most widely used algorithm for estimating material pyrolysis properties by optimization. However, Webster et al. [29,31] recently applied a stochastic hill climber (SHC) method to extract material pyrolysis properties from cone calorimeter experiments and concluded that the new approach provides better performance than GA. Similarly, Chaos et al. [32,33] determined that shuffled complex evolution (SCE) optimization [35] performs much better than GA for extracting material pyrolysis properties from FPA experiments.

Since optimization is an integral part of material pyrolysis property estimation, it is critical to determine which of the available optimization methods provides the best performance for typical material pyrolysis 
property estimation applications in terms of efficiency (how quickly it converges to a solution) and accuracy (how close the converged solution is to the global optimum). The latter is particularly important. In order to do this, three new optimization algorithms have recently been added to the pyrolysis model Gpyro [3] (as of Version 0.716). This now provides the user with four different choices of optimization algorithms for material pyrolysis property estimation:

1. Vanilla genetic algorithm (GA) [3]

2. Hybrid genetic algorithm/simulated annealing (GASA) [3]

3. Stochastic hill climber (SHC) $[29,31]$

4. Shuffled complex evolution (SCE) $[32,33,35]$

Details of these algorithms are available in the Gpyro technical reference [3] and Refs. [29,31-33,35] and are not repeated here. All but SHC have been parallelized using message passing interface (MPI), making it possible to conduct a typical material pyrolysis property estimation run requiring tens of thousands of trial solutions in a few hours on a 16-core computer cluster (circa 2010).

Although these algorithms have already been applied to material pyrolysis property estimation by others, their incorporation within Gpyro makes it possible to compare their relative performance for identical problems using an identical pyrolysis model formulation. Here, each of these algorithms is applied to a prototypical, but challenging, material pyrolysis property estimation problem to determine which algorithm provides the best performance in terms of efficiency and accuracy.

Table 2. Representative contributions to the field of material pyrolysis property estimation.

\begin{tabular}{ccllll}
\hline Ref. & Year & Authors & Type & Experiments & Materials \\
\hline$[9]$ & 1998 & de Ris \& Yan & Excel & FPA & Particle board, plywood \\
{$[10]$} & 1999 & Kanevce et al. & NR/SD & Custom & Phenolic composite \\
{$[11]$} & 2001 & Şahin et al. & GA & TGA & Ammonium pentaborate \\
{$[12]$} & 2005 & Zhao \& Dembsey & Excel & FPA & Wood, FRP \\
{$[13]$} & 2005 & Theuns et al. & DSM & Slab & Particle board \\
{$[14]$} & 2005 & Rein et al. & GA & TGA & PU foam \\
{$[15]$} & 2006 & Rein et al. & GA & TGA & PU foam \\
{$[16]$} & 2006 & Lautenberger et al. & GA & Cone, TGA & Wood, PP \\
{$[17]$} & 2006 & Lee & Manual & Cone & Wood, PMMA, Foam \\
{$[18]$} & 2007 & Lautenberger & GA & Cone, TGA & Wood, PMMA, PU, intumesc. \\
{$[19]$} & 2008 & Matala & GA, Manual & Cone, TGA & Wood, PMMA, PVC \\
{$[20]$} & 2008 & Saha et al. & HGA & TGA & PET, LDPE, PP \\
{$[21]$} & 2008 & Reddy et al. & HGA & TGA & PP \\
{$[22]$} & 2008 & Matala et al. & GA & TGA & Wood, PVC, PMMA \\
{$[23]$} & 2008 & Lautenberger et al. & GA & TGA, FPA & Polyester composite \\
{$[24]$} & 2009 & Lautenberger et al. & GA & Cone & PMMA, PE \\
{$[25]$} & 2009 & Lautenberger \& Pello & GA & Cone, TGA & Wood, PMMA, PU, intumesc. \\
{$[26]$} & 2009 & Lautenberger \& Pello & GA & Cone & Wood \\
{$[27]$} & 2009 & Bustamante & GA & TGA, Cone & PU \\
{$[28]$} & 2009 & Kim et al. & GA & TGA, FPA & Polyester composite \\
{$[29]$} & 2009 & Webster & SHC, GA & Cone & Carpet, FRP, phenolic panel \\
{$[30]$} & 2009 & Matala et al. & GA & TGA, Cone & PVC, PMMA, power cable \\
{$[31]$} & 2010 & Webster et al. & SHC, GA & Cone & Carpet \\
{$[32]$} & 2010 & Chaos et al. & SCE, GA & FPA & Corrugated, CPVC \\
{$[33]$} & 2010 & Chaos et al. & SCE, GA & FPA & PMMA, corrugated, CPVC \\
\hline DSM & Dow & Gill & & &
\end{tabular}

DSM: Downhill simplex method

Excel: Microsoft Excel 'solver' function

GA: Genetic algorithm

NR/SD: Newton-Raphson/steepest descent (modified Marquardt)

SHC: Stochastic hill climber

SCE: Shuffled complex evolution 


\section{SYNTHETIC EXPERIMENTAL DATA}

Optimization algorithms for material pyrolysis property estimation are assed here using a set of synthetic experimental data in lieu of actual experimental data. This makes it possible to determine how closely each optimization algorithm can reproduce the actual material pyrolysis properties that were used to generate the synthetic experimental data. Optimization methods have already been widely used to estimate material pyrolysis properties of real world materials, and it is not the intent of this work to demonstrate that this can be done, but rather to determine the optimization algorithms that are best suited for this purpose.

Synthetic experimental data are generated for the three-component, two reaction paradigm described in Equations 1-10 using the material pyrolysis properties listed in Table 3. Material thickness is $8 \mathrm{~mm}$ and the back and the back face is perfectly insulated. A constant convective heat transfer coefficient of $10 \mathrm{~W} / \mathrm{m}^{2} \cdot \mathrm{K}$ is assumed. Mass loss rate, surface temperature, and back face temperature are used as optimization targets for external heat flux levels of $25 \mathrm{~kW} / \mathrm{m}^{2}$ and $50 \mathrm{~kW} / \mathrm{m}^{2}$ (Fig. 1). The minimum and maximum values used to bound the range of allowable values in the optimization process is also indicated in Table 3 .

Table 3. Material pyrolysis properties used to generate synthetic experimental data.

\begin{tabular}{cccccc}
\hline$\#$ & Property & Units & $\begin{array}{c}\text { Target } \\
\text { value }\end{array}$ & $\begin{array}{c}\text { Minimum } \\
\text { value }\end{array}$ & $\begin{array}{c}\text { Maximum } \\
\text { value }\end{array}$ \\
\hline 1 & $k_{A}$ & $\mathrm{~W} / \mathrm{m} \cdot \mathrm{K}$ & 0.200 & 0.050 & 1.000 \\
2 & $c_{A}$ & $\mathrm{~J} / \mathrm{kg} \cdot \mathrm{K}$ & 1500 & 1000 & 4000 \\
3 & $\varepsilon_{A}$ & - & 0.650 & 0.500 & 1.000 \\
4 & $k_{B}$ & $\mathrm{~W} / \mathrm{m} \cdot \mathrm{K}$ & 0.150 & 0.05 & 1.00 \\
5 & $c_{B}$ & $\mathrm{~J} / \mathrm{kg}-\mathrm{K}$ & 1500 & 1000 & 4000 \\
6 & $\rho_{B}$ & $\mathrm{~kg} / \mathrm{m}^{3}$ & 200 & 100 & 400 \\
7 & $\varepsilon_{B}$ & - & 0.950 & 0.700 & 1.000 \\
8 & $k_{C}$ & $\mathrm{~W} / \mathrm{m} \cdot \mathrm{K}$ & 0.100 & 0.050 & 1.000 \\
9 & $c_{C}$ & $\mathrm{~J} / \mathrm{kg} \cdot \mathrm{K}$ & 1500 & 1000 & 4000 \\
10 & $\rho_{C}$ & $\mathrm{~kg} / \mathrm{m}^{3}$ & 50.0 & 20.0 & 100.0 \\
11 & $\varepsilon_{C}$ & - & 0.900 & 0.700 & 1.000 \\
12 & $\log Z_{1}$ & $\operatorname{log~s} \mathrm{s}^{-1}$ & 8.70 & 7.00 & 11.00 \\
13 & $E_{1}$ & $\mathrm{~kJ} / \mathrm{mol}$ & 130.0 & 100.0 & 170.0 \\
14 & $n_{1}$ & - & 1.00 & 0.50 & 2.00 \\
15 & $\log \Delta H_{\text {voll }}$ & $\log \mathrm{J} / \mathrm{kg}$ & 5.78 & 4.00 & 6.30 \\
16 & $\log Z_{2}$ & $\log \mathrm{s}^{-1}$ & 10.70 & 8.00 & 12.00 \\
17 & $E_{2}$ & $\mathrm{~J} / \mathrm{mol}$ & 175.0 & 140.0 & 210.0 \\
18 & $n_{2}$ & - & 1.00 & 0.50 & 2.00 \\
19 & $\log \Delta H_{\text {vol2 }}$ & $\log \mathrm{J} / \mathrm{kg}$ & 5.00 & 4.00 & 6.30 \\
\hline
\end{tabular}

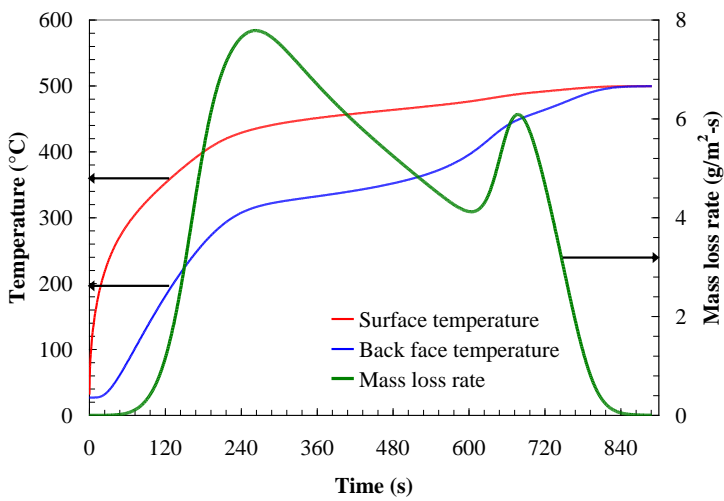

(a)

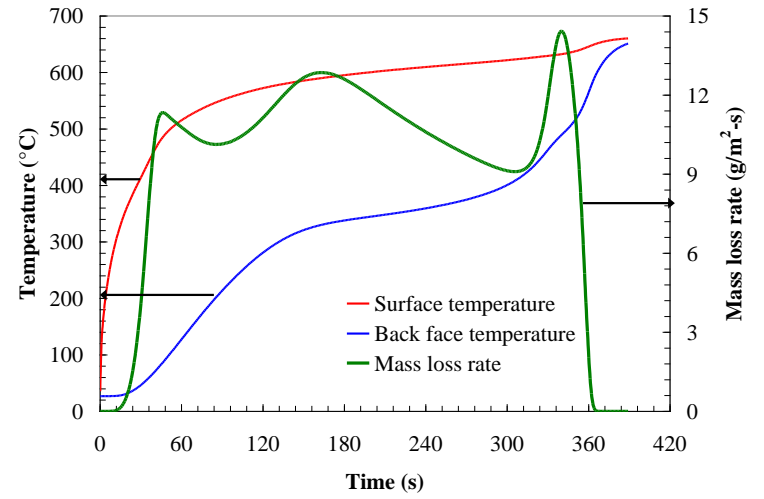

(b)

Fig. 1. Synthetic experimental data: (a) $25 \mathrm{~kW} / \mathrm{m}^{2}$; (b) $50 \mathrm{~kW} / \mathrm{m}^{2}$. 


\section{MATERIAL PYROLYSIS PROPERTY ESTIMATION FROM SYNTHETIC DATA}

Efficiency (how quickly an optimization algorithm converges to a solution) and accuracy (how close the ultimate solution is to the global optimum) can be assessed from fitness evolution, i.e. a plot of fitness (a quantitative measure of how closely the best trial solution found so far matches the true solution) as a function of the number of function evaluations (the number of calls to the pyrolysis algorithm with different trial solutions/material pyrolysis properties). It should be pointed out that other workers have used fitness functions based on error minimization wherein lower fitness values correspond to better solutions, but the current work uses the fitness function given in the Gpyro technical reference [3] where a higher value of fitness corresponds to a better solution. A higher fitness corresponds to a better solution (set of material pyrolysis properties), but the numerical fitness value is arbitrary.

Figure $2 \mathrm{a}$ shows the fitness evolution for GA optimization as a function of the population size. A population size of around 250 is optimal, with the fitness reaching a peak value of $\sim 1500$ after 25,000 function evaluations. However, no additional improvement occurs through 120,000 total function evaluations, meaning that the solution has converged.

Figure $2 b$ shows the GASA fitness evolution. Although this algorithm initially converges less rapidly than the vanilla GA, except for a population size of 125, it does ultimately reach higher fitness levels than the vanilla GA. The highest fitness $(\sim 2,000)$ is obtained for a population size of 1,000 , and it is possible that higher fitness values could have been reached with additional trial solutions.

Figure 2c shows the SHC fitness evolution. This algorithm is characterized by very rapid initial convergence than either GA or GASA. For the optimal case with a mutation probability of 0.25 and a mutation severity of 1 , after $\sim 30,000$ function evaluations the fitness reached a comparable level to that reached by GASA after $\sim 100,000$ function evaluations. Therefore, when properly optimized, the SHC algorithm is more efficient than GA or GASA as implemented in Gpyro. However it is not clear whether SHC or GA/GASA is more accurate since comparable fitness levels were reached for both.

Figure $2 \mathrm{~d}$ shows the SCE fitness evolution. While initial convergence is not as rapid as with SHC, a higher ultimate fitness level is reached. To assess the effect of the random number generator seed, ten different instances were run using different random number seeds. Each trace in Fig. 2d corresponds to one of these instances. It can be seen that the fitness evolution follows a similar pattern in all cases. This suggests that the SCE converges to a 'good' solution occurs regardless of the initial guess or sequence of random numbers. Furthermore, as will be shown below, all ten instances converged to the same 'good' solution the global optimum.

The four optimization methods are compared in Fig. 3 by plotting the fitness evolution from each method that gave the highest ultimate fitness. It can be seen that SCE provides the highest final fitness, followed by GASA and SHC which reached similar ultimate fitness levels. Vanilla GA reached the lowest fitness level. In terms of efficiency, SHC converges to a higher fitness level than any of the other methods over the first $\sim 5,000$ function evaluations. However, by $\sim 15,000$ function evaluations, SCE reaches a higher fitness than that reached by SHC after 50,000 function evaluations, GASA after 100,000 function evaluations, and GA after 100,000 function evaluations.

Table 4 shows the material pyrolysis properties corresponding to the best solution determined by each of the four optimization methods. Also shown are the target values and the percentage error of each method for each variable. The SCE approach has the lowest average percentage error of the four methods, followed by GASA, GA, and then SHC.

Table 5 presents statistics from the 10 independent SCE trials that were conducted using different random number seeds. These statistics include the minimum, maximum, and average value of each parameter found over the 10 trials as well as the absolute and normalized standard deviation. It is shown that the solution always converges to a nominally identical parameter set corresponding to the global optimum. For all parameters, the normalized standard deviation is less than $1.5 \%$, and for all parameters excluding $c_{C}$, it is less than $1 \%$. This indicates that for this prototypical but challenging test problem (involving simultaneous optimization of 19 parameters) SCE consistently converges to a unique solution corresponding to the global optimum. 

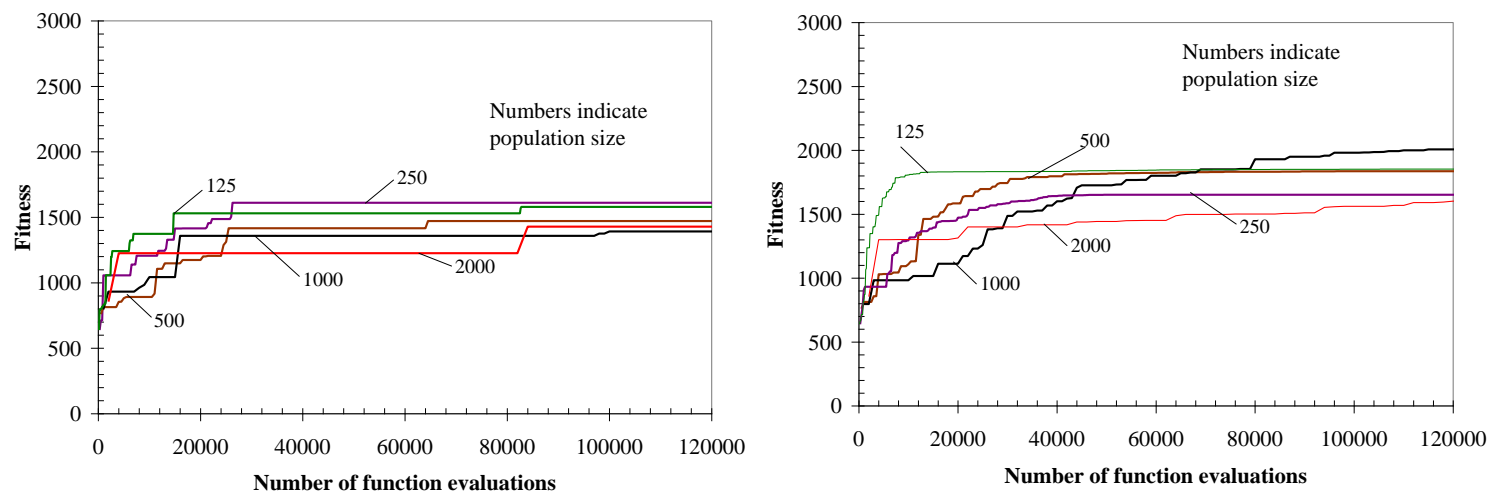

(a)

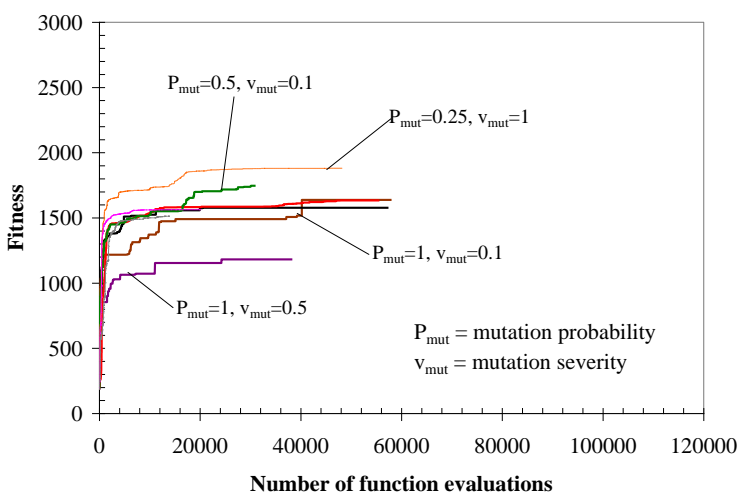

(c)

(b)

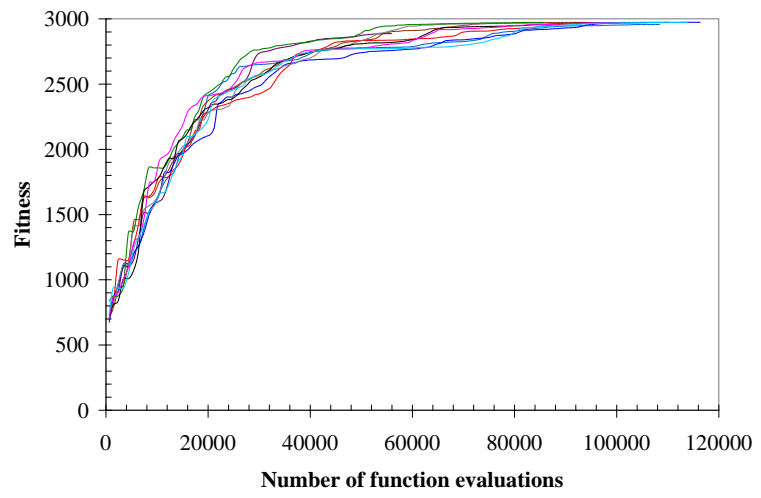

(d)

Fig. 2. Fitness evolution: (a) genetic algorithm (GA); (b) genetic algorithm with simulated annealing

(GASA); (c) stochastic hill climber (SHC); (d) shuffled complex evolution (SCE).

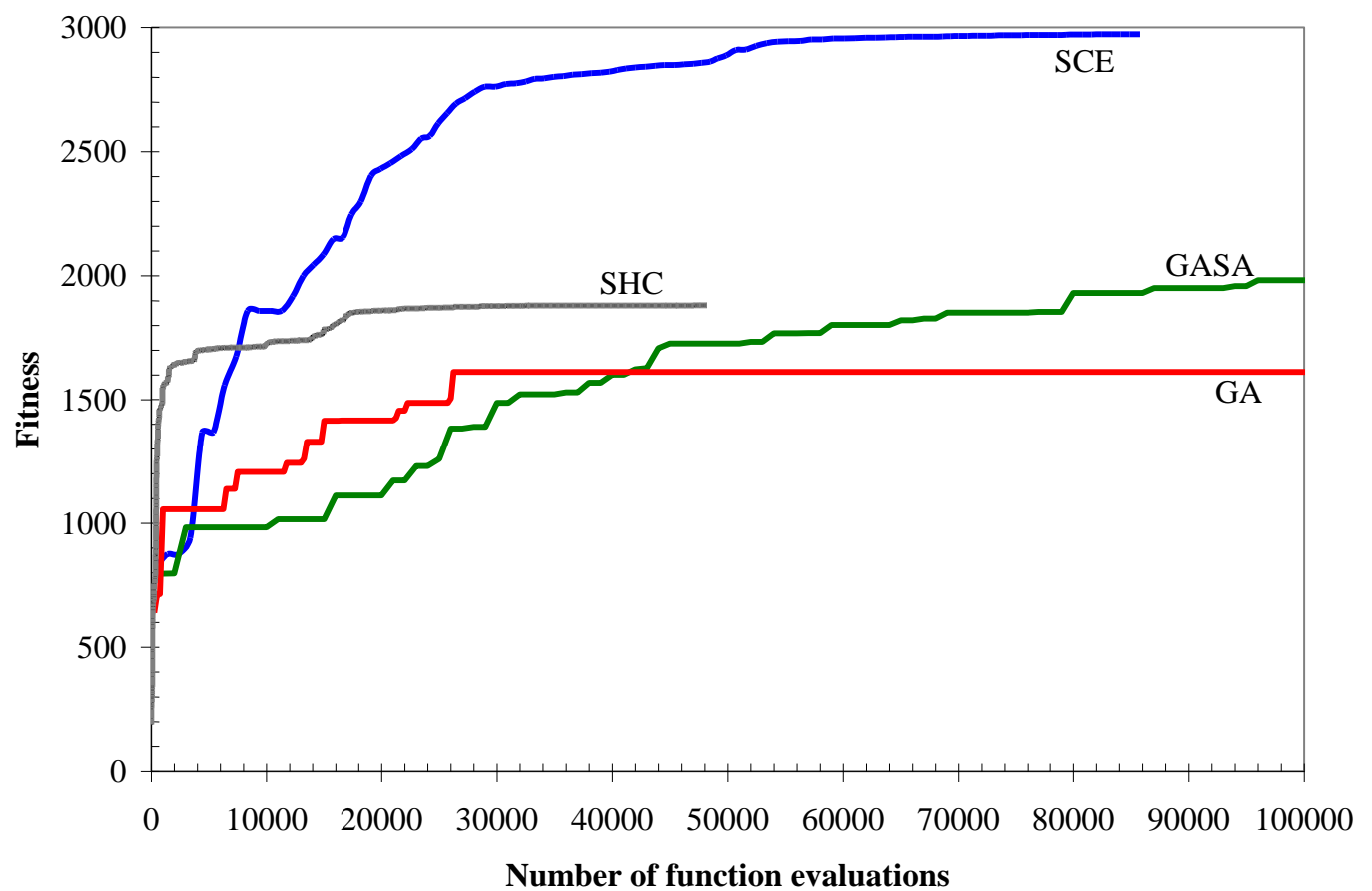

Fig. 3. Comparison of GA, GASA, SHC, and SCE optimization methods. 
Table 4. Optimal solutions located by SCE, CA, GASA, and SHC.

\begin{tabular}{|c|c|c|c|c|c|c|c|c|c|}
\hline \multirow{2}{*}{ Variable } & \multirow[b]{2}{*}{ Target } & \multicolumn{4}{|c|}{ Best solution values } & \multicolumn{4}{|c|}{ Best solution percentage error } \\
\hline & & SCE & GA & GASA & SHC & SCE & GA & GASA & SHC \\
\hline$k_{A}$ & 0.200 & 0.201 & 0.253 & 0.224 & 0.236 & 0.35 & 26.56 & 12.03 & 18.03 \\
\hline$c_{A}$ & 1500 & 1504 & 1979 & 1666 & 1757 & 0.30 & 31.91 & 11.05 & 17.17 \\
\hline$\varepsilon_{A}$ & 0.650 & 0.652 & 0.941 & 0.706 & 0.744 & 0.35 & 44.77 & 8.66 & 14.45 \\
\hline$k_{B}$ & 0.150 & 0.152 & 0.111 & 0.081 & 0.003 & 1.27 & -26.33 & -46.17 & -98.04 \\
\hline$c_{B}$ & 1500 & 1532 & 1877 & 2842 & 1436 & 2.12 & 25.12 & 89.44 & -4.28 \\
\hline$\rho_{B}$ & 200 & 200 & 177 & 217 & 210 & -0.02 & -11.42 & 8.57 & 4.84 \\
\hline$\varepsilon_{B}$ & 0.950 & 0.955 & 0.795 & 0.876 & 0.863 & 0.53 & -16.31 & -7.75 & -9.13 \\
\hline$k_{C}$ & 0.100 & 0.101 & 0.117 & 0.109 & 0.123 & 1.07 & 16.79 & 9.30 & 23.31 \\
\hline$c_{C}$ & 1500 & 1542 & 2182 & 1675 & 728 & 2.77 & 45.49 & 11.64 & -51.44 \\
\hline$\rho_{C}$ & 50.0 & 49.5 & 47.9 & 53.6 & 79.3 & -0.91 & -4.27 & 7.22 & 58.63 \\
\hline$\varepsilon_{C}$ & 0.900 & 0.902 & 0.931 & 0.873 & 0.982 & 0.22 & 3.49 & -3.04 & 9.13 \\
\hline $\log Z_{1}$ & 8.70 & 8.72 & 9.75 & 8.88 & 8.32 & 0.28 & 12.09 & 2.07 & -4.38 \\
\hline$E_{1}$ & 130.0 & 130.3 & 141.7 & 130.9 & 125.7 & 0.23 & 9.02 & 0.73 & -3.34 \\
\hline$n_{1}$ & 1.00 & 1.00 & 1.40 & 1.17 & 1.83 & -0.04 & 40.45 & 16.55 & 83.12 \\
\hline $\log \Delta H_{v o l 1}$ & 5.78 & 5.78 & 5.67 & 5.62 & 5.73 & 0.07 & -1.96 & -2.71 & -0.87 \\
\hline $\log Z_{2}$ & 10.70 & 10.67 & 10.50 & 10.37 & 10.15 & -0.29 & -1.87 & -3.08 & -5.16 \\
\hline$E_{2}$ & 175.0 & 174.5 & 172.9 & 167.7 & 140.3 & -0.26 & -1.20 & -4.17 & -19.84 \\
\hline$n_{2}$ & 1.00 & 1.00 & 1.41 & 1.22 & 0.63 & 0.02 & 41.24 & 22.31 & -37.28 \\
\hline $\log \Delta H_{v o l 2}$ & 5.00 & 5.00 & 4.83 & 4.32 & 5.76 & 0.05 & -3.47 & -13.57 & 15.29 \\
\hline & & & & Absolut & erage: & 0.59 & 19.14 & 14.74 & 25.14 \\
\hline
\end{tabular}

Table 5. Statistics of optimal solution determined by SCE over 10 independent trials.

\begin{tabular}{ccccccc}
\hline Variable & Target & Minimum & Maximum & Average & $\begin{array}{c}\text { Absolute } \\
\text { std. dev. }\end{array}$ & $\begin{array}{c}\text { Normalized } \\
\text { std. dev. }(\%)\end{array}$ \\
\hline$k_{A}$ & 0.200 & 0.200 & 0.202 & 0.201 & 0.001 & 0.30 \\
$c_{A}$ & 1500 & 1496 & 1511 & 1504 & 4 & 0.29 \\
$\varepsilon_{A}$ & 0.650 & 0.649 & 0.654 & 0.652 & 0.001 & 0.22 \\
$k_{B}$ & 0.15 & 0.152 & 0.154 & 0.152 & 0.001 & 0.41 \\
$c_{B}$ & 1500 & 1501 & 1555 & 1531 & 15 & 0.98 \\
$\rho_{B}$ & 200 & 199 & 201 & 200 & 0.4 & 0.22 \\
$\varepsilon_{B}$ & 0.950 & 0.951 & 0.964 & 0.955 & 0.004 & 0.38 \\
$k_{C}$ & 0.100 & 0.100 & 0.103 & 0.101 & 0.001 & 0.74 \\
$c_{C}$ & 1500 & 1517 & 1584 & 1545 & 22 & 1.49 \\
$\rho_{C}$ & 50.0 & 49.5 & 49.7 & 49.6 & 0.0 & 0.09 \\
$\varepsilon_{C}$ & 0.900 & 0.900 & 0.906 & 0.902 & 0.002 & 0.18 \\
$\log Z_{1}$ & 8.70 & 8.70 & 8.84 & 8.72 & 0.04 & 0.50 \\
$E_{1}$ & 130.0 & 130.0 & 131.7 & 130.3 & 0.5 & 0.39 \\
$n_{1}$ & 1.00 & 0.99 & 1.02 & 1.00 & 0.01 & 0.68 \\
$\log \Delta H_{\text {vol } 1}$ & 5.78 & 5.78 & 5.79 & 5.78 & 0.00 & 0.03 \\
$\log Z_{2}$ & 10.70 & 10.59 & 10.78 & 10.67 & 0.05 & 0.49 \\
$E_{2}$ & 175.0 & 173.4 & 176.2 & 174.6 & 0.7 & 0.42 \\
$n_{2}$ & 1.00 & 1.00 & 1.00 & 1.00 & 0.00 & 0.09 \\
$\log \Delta H_{\text {vol }}$ & 5.00 & 5.00 & 5.01 & 5.00 & 0.00 & 0.05 \\
\hline
\end{tabular}

\section{DISCUSSION AND CONCLUSIONS}

Due to its balance between efficiency and accuracy, shuffled complex evolution (SCE) optimization is recommended for general use in material pyrolysis property estimation. As implemented in Gpyro's companion material pyrolysis property estimation program, SCE consistently performs better than the 
genetic algorithm, genetic algorithm/simulated annealing, and stochastic hill climber algorithms for the test problem involving synthetic experimental data considered here. These findings are consistent with Chaos et al. $[32,33]$ who concluded that SCE offers advantages over GA for material pyrolysis property estimation.

Early studies on material pyrolysis property estimation using optimization pointed out that the problem is ill-posed, i.e. multiple sets of different material pyrolysis properties may provide equally good fits to experimental data so a unique solution does not exist. However, for the test case involving synthetic experimental data considered here, SCE consistently converges to the same solution and is capable of reproducing material pyrolysis properties within $\sim 1 \%$ of the actual values used to generate the synthetic data set. This suggests that SCE is capable of determining a unique set of material pyrolysis properties that correspond to the globally optimal solution.

\section{ACKNOWLEDGMENTS}

This work was supported by the National Science Foundation under Grant 0730556 . The authors thank Rob Webster for discussions regarding the stochastic hill climber algorithm.

\section{REFERENCES}

[1] McGrattan, K., McDermott, R., Hostikka, S., and Floyd, J., "Fire Dynamics Simulator (Version 5) User's Guide," NIST Special Publication 1019-5, National Institute of Standards and Technology, Gaithersburg, MD, 2010.

[2] Stoliarov, S.I. and Lyon, R.E., "Thermo-Kinetic Model of Burning," Federal Aviation Administration, DOT/FAA/AR-TN08/17, May 2008.

[3] Gpyro, A Generalized Pyrolysis Model for Combustible Solids, http://code.google.com/p/gpyro

[4] Kim, E., Dembsey, N., and Janssens, M., "SFPE Engineering Guide for Estimating Material Pyrolysis Properties for Fire Modeling," Draft 2 - May 1, 2010.

[5] FireFOAM, http://code.google.com/p/firefoam-dev

[6] Lattimer, B.Y. and Ouellette, J., (2006) Properties of composite materials for thermal analysis involving fires, Composites: Part A 37: 1068-1081, http://dx.doi.org/10.1016/j.compositesa.2005.01.029

[7] Stoliarov, S.I., Crowley, S., Lyon, R.E., and Linteris, G.T., (2009) Prediction of the burning rates of non-charring polymers, Combustion and Flame 156: 1068-1083, http://dx.doi.org/10.1016/j.combustflame.2008.11.010

[8] Stoliarov, S.I., Crowley, S., Walters, R.N., and Lyon, R.E., (2010) Prediction of the burning rates of charring polymers, Combustion and Flame, in press, http://dx.doi.org/10.1016/j.combustflame.2010.03.011

[9] de Ris, J.L. and Yan, Z., Modeling ignition and pyrolysis of solid fuels, Proceedings of the Fifth International Conference on Fire and Materials, 1998, pp. 111-121.

[10] Kanevce, L.P., Kanevce, G.H., and Angelevski, Z.Z., Comparison of two kinds of experiments for estimation of thermal properties of ablative composite, Inverse Problems in Engineering: Theory and Practice, Third International Conference on Inverse Problems in Engineering, 1999, pp. 1-7.

[11] Şahin, Ö, Özdemir, M., Aslanoğlu, M., Gürbüz Beker, Ü., (2001) Calcination Kinetics of Ammonium Pentaborate Using the Coats-Redfern and Genetic Algorithm Method by Thermal Analysis, Industrial \& Engineering Chemistry Research 40: 1465-1470, http://dx.doi.org/10.1021/ie000690f

[12] Zhao, L. and Dembsey, N.A., "Uncertainty effects on measurement of fire characteristics of material systems," Proceedings of the Ninth International Conference on Fire and Materials, 2005, pp. 37-49. 
[13] Theuns, E., Merci, B., Vierendeels, J., and Vandevelde, P., (2005) Critical evaluation of an integral model for the pyrolysis of charring materials Fire Safety Journal 40: 121-140, http://dx.doi.org/10.1016/j.firesaf.2004.09.003

[14] Rein, G., Lautenberger, C., and Fernandez-Pello, A.C., Using Genetic Algorithms to Derive the Parameters of Solid-Phase Combustion from Experiments, 20th International Colloquium on the Dynamics of Explosions and Reactive Systems, 2005.

[15] Rein, G., Lautenberger, C., Fernandez-Pello, A.C., Torero, J.L. and Urban, D.L., (2006) Application of Genetic Algorithms and Thermogravimetry to Determine the Kinetics of Polyurethane Foam in Smoldering Combustion, Combustion and Flame 146: 95-108, http://dx.doi.org/10.1016/j.combustflame.2006.04.013

[16] Lautenberger, C., Rein, G., and Fernandez-Pello, A.C., (2006) The Application of a Genetic Algorithm to Estimate Material Properties for Fire Modeling from Bench-Scale Fire Test Data Fire Safety Journal 41: 204-214, http://dx.doi.org/10.1016/j.firesaf.2005.12.004

[17] Lee, S.H., "Material Property Estimation Method Using a Thermoplastic Pyrolysis Model," MS Thesis, Department of Fire Protection Engineering, Worcester Polytechnic Institute, Worcester, MA (2006).

[18] Lautenberger, C., "A Generalized Pyrolysis Model for Combustible Solids," PhD Dissertation, Department of Mechanical Engineering, University of California, Berkeley, CA (2007).

[19] Matala, A., "Estimation of solid phase reaction parameters for fire simulation," MS Thesis, Helsinki University of Technology (2008).

[20] Saha, B., Reddy, P.K., and Ghosal, A.K., (2008) Hybrid genetic algorithm to find the best model and the globally optimized overall kinetics parameters for thermal decomposition of plastics, Chemical Engineering Journal 138: 20-29, http://dx.doi.org/10.1016/j.cej.2007.05.024

[21] Reddy, P.K., Chowlu, A.C.K., and Ghosal, A.K., (2008) Hybrid genetic algorithm and model-free coupled direct search methods for kinetics of nanocrystalline ZSM-5-catalyzed decomposition of PP, Applied Catalysis A: General 351: 195-203, http://dx.doi.org/10.1016/j.apcata.2008.09.016

[22] Matala, A., Hostikka, S., and Mangs, J., (2008) Estimation of Pyrolysis Model Parameters for Solid Materials Using Thermogravimetric Data, Fire Safety Science 9: 1213-1224, http://dx.doi.org/10.3801/IAFSS.FSS.9-1213

[23] Lautenberger, C., Kim, E., Dembsey, N., and Fernandez-Pello, C., (2008) The Role of Decomposition Kinetics in Pyrolysis Modeling - Application to a Fire Retardant Polyester Composite, Fire Safety Science 9: 1201-1212, http://dx.doi.org/10.3801/IAFSS.FSS.9-1201

[24] Lautenberger, C., Wong, W., Dembsey, N., Coles, A., and Fernandez-Pello, C., Large-Scale Turbulent Flame Spread Modeling with FDS5 on Charring and Noncharring Materials, Proceedings of the Eleventh International Conference on Fire and Materials, 2009.

[25] Lautenberger, C. and Fernandez-Pello, A.C., (2009) Generalized Pyrolysis Model for Combustible Solids, Fire Safety Journal 44: 819-839, http://dx.doi.org/10.1016/j.firesaf.2009.03.011

[26] Lautenberger, C. and Fernandez-Pello, A.C., (2009) A Model for the Oxidative Pyrolysis of Wood, Combustion and Flame 156: http://dx.doi.org/10.1016/j.combustflame.2009.04.001

[27] Bustamante Valencia, L., Experimental and numerical investigation of the thermal decomposition of materials at three scales: application to polyether polyurethane foam used in upholstered furniture, $\mathrm{PhD}$ Thesis, 2009.

[28] Kim, E., Lautenberger, C., and Dembsey, N., Property Estimation for Pyrolysis Modeling Applied to Polyester FRP Composites with Different Glass Contents, Composites \& Polycon 2009, American Composites Manufacturers Association, 2009. 
[29] Webster, R.D., Pyrolysis Model Parameter Optimization Using a Customized Stochastic HillClimber Algorithm and Bench-Scale Fire Test Data, MS Thesis, Department of Fire Protection Engineering, University of Maryland, 2009.

[30] Matala, A., Hostikka, S., and Mangs, J., Estimation of pyrolysis model parameters for condenced phase materials, Chapter 31.2 in VTT Research Notes 2466, SAFIR2010 The Finnish Research Programme on Nuclear Power Plant Safety 2007-2010, Interim Report, 2009.

[31] Webster, R., Lázaro, M., Alvear, D., Capote, J., and Trouvé, A., Limitations in Current Parameter Estimation Techniques for Pyrolysis Modeling, Sixth Fire and Explosion Hazards Seminar (FEH6), 2010.

[32] Chaos, M., Khan, M.M., Krishnamoorthy, N., de Ris, J.L., and Dorofeev, S.B., Bench-scale flammability experiments: determination of material properties using pyrolysis models for use in CFD fire simulations, Interflam 2010, 2010, pp. 697-708.

[33] Chaos, M., Khan, M.M., Krishnamoorthy, N., de Ris, J.L., and Dorofeev, S.B., (2010) Evaluation of optimization schemes and determination of solid fuel properties for CFD fire models using bench-scale pyrolysis tests, Proceedings of the Combustion Institute 33, in press, http://dx.doi.org/10.1016/j.proci.2010.07.018

[34] Kung, H.-C., (1972) A mathematical model of wood pyrolysis, Combustion and Flame 18: 185195, http://dx.doi.org/10.1016/S0010-2180(72)80134-2

[35] Duan, Q.Y., Gupta, V.K., and Sorooshian, S., (1993) Shuffled Complex Evolution Approach for Effective and Efficient Global Minimization, Journal of Optimization Theory and Applications 76: 501-521, http://dx.doi.org/10.1007/BF00939380 